Why do the Heavy-Atom Analogues of Acetylene $\mathrm{E}_{2} \mathrm{H}_{2}(\mathrm{E}=\mathrm{Si}-\mathrm{Pb})$ Exhibit Unusual Structures?

Matthias Lein, Andreas Krapp and Gernot Frenking*

Supporting Information (4 Tables and complete references 24 and 29) 
Table S1. Energy Decomposition Analysis of $\mathrm{E}_{2} \mathrm{H}_{2}$ Isomer D2 (E = Si, Ge, $\mathrm{Sn}, \mathrm{Pb}$ ) Using $\mathrm{EH}$ as Fragments. The Energy Values are Given in kcal/mol. The $\mathrm{E}_{2} \mathrm{H}_{2}$ Structures were Optimized with the E-E Distance Frozen at the Value in A.

\begin{tabular}{lllll}
\hline Term & $\mathrm{Si}_{2} \mathrm{H}_{2}$ & $\mathrm{Ge}_{2} \mathrm{H}_{2}$ & $\mathrm{Sn}_{2} \mathrm{H}_{2}$ & $\mathrm{~Pb}_{2} \mathrm{H}_{2}$ \\
\hline$\Delta \mathrm{E}_{\text {int }}$ & -30.23 & -29.79 & -28.66 & -27.69 \\
$\Delta \mathrm{E}_{\text {Pauli }}$ & 226.17 & 203.52 & 165.59 & 146.20 \\
$\Delta \mathrm{E}_{\text {elstat }}{ }^{\mathrm{a}}$ & $-139.57(54.4 \%)$ & $-138.44(59.3 \%)$ & $-122.71(63.2 \%)$ & $-113.52(65.3 \%)$ \\
$\Delta \mathrm{E}_{\text {orb }}{ }^{\mathrm{a}}$ & $-116.83(45.6 \%)$ & $-94.86(40.7 \%)$ & $-71.55(36.8 \%)$ & $-60.37(34.7 \%)$ \\
$\Delta \mathrm{E}\left(\mathrm{A}^{\prime}\right)^{\mathrm{b}}$ & $-116.77(99.9 \%)$ & $-94.46(99.6 \%)$ & $-71.09(99.4 \%)$ & $-59.85(99.1 \%)$ \\
$\Delta \mathrm{E}\left(\mathrm{A}^{\prime \prime}\right)^{\mathrm{b}}$ & -0.06 & -0.40 & -0.46 & -0.53 \\
\hline
\end{tabular}

a The value in parentheses gives the percentage contribution to the total attractive interactions $\Delta \mathrm{E}_{\text {elstat }}$ $+\Delta \mathrm{E}_{\text {orb }}$.

${ }^{\mathrm{b}}$ The value in parentheses gives the percentage contribution to the total orbital interactions $\Delta \mathrm{E}_{\text {orb }}$. 
Table S2. Energy Decomposition Analysis of $\mathrm{E}_{2} \mathrm{H}_{2}$ Isomer D1 (E = Si, Ge, Sn, Pb) Using $\mathrm{EH}$ as Fragments. The Energy Values are Given in kcal/mol. Single-point Calculations of $\mathrm{E}_{2} \mathrm{H}_{2}$ with the E-E, E-H Distances and the E-E-H Angle were Taken from $\mathbf{B}$ Isomer.

\begin{tabular}{lllll}
\hline Term & $\mathrm{Si}_{2} \mathrm{H}_{2}$ & $\mathrm{Ge}_{2} \mathrm{H}_{2}$ & $\mathrm{Sn}_{2} \mathrm{H}_{2}$ & $\mathrm{~Pb}_{2} \mathrm{H}_{2}$ \\
\hline$\Delta \mathrm{E}_{\text {int }}$ & -48.73 & -33.55 & -26.12 & -19.72 \\
$\Delta \mathrm{E}_{\text {Pauli }}$ & 258.42 & 231.91 & 170.52 & 116.75 \\
$\Delta \mathrm{E}_{\text {elstat }}{ }^{\mathrm{a}}$ & $-123.42(40.2 \%)$ & $-126.78(47.8 \%)$ & $-101.60(51.7 \%)$ & $-73.33(53.7 \%)$ \\
$\Delta \mathrm{E}_{\text {orb }}{ }^{\mathrm{a}}$ & $-183.71(59.8 \%)$ & $-138.69(52.2 \%)$ & $-95.04(48.3 \%)$ & $-63.15(46.3 \%)$ \\
$\Delta \mathrm{E}\left(\mathrm{A}^{\prime}\right)^{\mathrm{b}}$ & $-143.43(78.1 \%)$ & $-103.54(74.7 \%)$ & $-68.12(71.7 \%)$ & $-40.38(63.9 \%)$ \\
$\Delta \mathrm{E}\left(\mathrm{A}^{\prime \prime}\right)^{\mathrm{b}}$ & $-40.28(21.9 \%)$ & $-35.15(25.3 \%)$ & $-26.92(28.3 \%)$ & $-22.77(36.1 \%)$ \\
\hline
\end{tabular}

a The value in parentheses gives the percentage contribution to the total attractive interactions $\Delta \mathrm{E}_{\text {elstat }}$ $+\Delta \mathrm{E}_{\text {orb }}$.

${ }^{\mathrm{b}}$ The value in parentheses gives the percentage contribution to the total orbital interactions $\Delta \mathrm{E}_{\text {orb }}$. 
Table S3. Calculated Absolute Energies (in Hartrees) and Relative Energies (in $\mathrm{kcal} / \mathrm{mol}$ ) of the $\mathrm{Si}_{2} \mathrm{H}_{2}$ Isomers $\mathbf{A}-\mathbf{I}(\mathbf{T})$ at the MRCI-SD/aug-cc-pVQZ and MRCI-SD(Q)/aug-cc-pVQZ Levels of Theory. The Values for a0 Give the Coefficients of the Hartree-Fock Reference Configuration in the MRCI Expansion.

\begin{tabular}{lccccc}
\hline Isomer & MRCI-SD & $\mathrm{E}_{\text {rel }}$ & $\mathrm{a} 0$ & MRCI-SD $(\mathrm{Q})$ & $\mathrm{E}_{\text {rel }}$ \\
\hline A & -579.225739 & 0.00 & 0.910167 & -579.244124 & 0.00 \\
B & -579.210484 & 9.57 & 0.898714 & -579.227837 & 10.22 \\
D2 & -579.161488 & 40.32 & 0.899292 & -579.178200 & 41.37 \\
D1 & -579.199778 & 16.29 & 0.871612 & -579.215681 & 17.85 \\
F & -579.158034 & 42.49 & 0.898322 & -579.173953 & 44.03 \\
G & -579.208463 & 10.84 & 0.896531 & -579.226580 & 11.01 \\
E & -579.166180 & 37.37 & 0.893831 & -579.182032 & 38.96 \\
C & -579.207041 & 11.73 & 0.900137 & -579.223486 & 12.95 \\
A(T) & -579.141195 & 53.05 & 0.909555 & -579.165940 & 49.06 \\
B(T) & -579.172950 & 33.13 & 0.915816 & -579.189931 & 34.01 \\
D(T) & -579.185465 & 25.27 & 0.903987 & -579.202590 & 26.06 \\
F(T) & -579.165545 & 37.77 & 0.891867 & -579.183031 & 38.34 \\
H(T) & -579.170284 & 34.80 & 0.898845 & -579.187276 & 35.67 \\
G(T) & -579.138789 & 54.56 & 0.910920 & -579.164224 & 50.14 \\
E(T) & -579.088377 & 86.20 & 0.856040 & -579.107189 & 85.93 \\
C(T) & -579.191069 & 21.76 & 0.921046 & -579.208256 & 22.51 \\
I(T) & -579.170582 & 34.61 & 0.912800 & -579.188531 & 34.89 \\
\hline
\end{tabular}


Table S4. Calculated Energies of the $\mathrm{E}_{2} \mathrm{H}_{2}$ Forms A - E (in Hartrees) of ADF at BP86/QZ4P. The Program Gives Only Relative Energies of the Molecules with Respect to the Energies of the Atoms.

\begin{tabular}{lcccccc}
\hline $\mathrm{E}$ & A & B & C & D1 & D2 & E \\
\hline $\mathrm{C}$ & - & - & - & - & - & -0.83566647 \\
$\mathrm{Si}$ & -0.50783625 & -0.49212019 & -0.48340284 & -0.47605401 & -0.43508475 & -0.44176921 \\
$\mathrm{Ge}$ & -0.46747420 & -0.45006212 & -0.44119501 & -0.43192511 & -0.40758219 & -0.37865701 \\
$\mathrm{Sn}$ & -0.42119093 & -0.39960403 & -0.39047666 & -0.38089957 & -0.37449437 & -0.31822014 \\
$\mathrm{~Pb}$ & -0.39995839 & -0.37283746 & -0.35683265 & -0.35058272 & $-0.35777119-0.25254951$ \\
\hline
\end{tabular}




\section{Complete References}

(24) Baerends, E.J.; J. Autschbach, J.A.; Berces, A.; Bo, C.; Boerrigter, P.M.; Cavallo, L.; Chong, D.P.; Deng, L.; Dickson, R.M.; Ellis, D.E.; Fan, L.; Fischer, T.H.; Fonseca Guerra, C.; van Gisbergen, S.J.A.; Groeneveld, J.A.; Gritsenko, O.V.; Grüning, M.; Harris, F.E.; van den Hoek, P.; Jacobsen, H.; van Kessel, G.; Kootstra, F.; van Lenthe, E.; Osinga, V.P.; Patchkovskii, S.; Philipsen, P.H.T.; Post, D.; Pye, C.C.; Ravenek, W.; Ros, P.; Schippen, P.R.T.; Schreckenbach, G.; Snijders, J.G.; Sola, M.; Swart, M.; Swerhone, D.; te Velde, G.; Vernooijs, G.; Versluis, L.; Visser, O.; Wezenbeek, E.; Wiesenekker, G.; Wolff, S.K.; Woo, T.K.; Ziegler, T. ADF 2003-01,; Scientific Computing \& Modelling NV: Amsterdam, The Netherlands (http://www.scm.com/), 2003.

(29) MOLPRO 2000 is a package of ab initio programs written by H. J. Werner, P. J. Knowles, M. Schütz, R. Lindh, P. Celani, T. Korona, G. Rauhut, F.R. Many, R. D. Amos, A. Bernhardsson, A. Berning, D. L. Cooper, M. J. O. Deegan, A. J. Dobbyn, F. Eckart, C. Hampel, G. Hetzer, A. W. Lloyd, S.J. McNicholas, W. Meyer, M. E. Mura, A. Nicklaß, P. Palmieri, R. Pitzer, U. Schumann, H. Stoll, A.J. Stone, R. Tarroni, and T. Thorsteinsson. 